\title{
Preventing Progression to Senile Dementia in Community-Dwelling Citizens of a Small Japanese Village
}

\author{
Kuniaki Otsuka*1,2 , Germaine Cornelissen ${ }^{2}$, Yutaka Kubo ${ }^{3}$, Tomoko Kikuchi ${ }^{3,4}$, Gaku Yamanaka ${ }^{3}$ \\ and Shougo Murakami ${ }^{5}$ \\ ${ }^{1}$ Executive Medical Center, Tokyo Women's Medical University, Japan \\ ${ }^{2}$ Halberg Chronobiology Center, University of Minnesota, USA
}

${ }^{3}$ Department of Medicine, Tokyo Women's Medical University, Medical Center East, Japan

${ }^{4}$ Cardiovascular Internal Medicine, Higashi Omiya General Hospital, Japan

${ }^{5}$ Department of Cardiovascular Medicine, Soseikai General Hospital, Japan

*Corresponding author: Kuniaki Otsuka, Executive Medical Center, Totsuka Royal Clinic, Tokyo Women’s Medical University, Tokyo, Japan.

To Cite This Article: Kuniaki Otsuka, Preventing Progression to Senile Dementia in Community-Dwelling Citizens of a Small Japanese Village. 2020 - 10(1). AJBSR.MS.ID.001462. DOI: 10.34297/AJBSR.2020.10.001462.

Received: 眥August 10, 2020; Published: 眥 August 20, 2020

\section{Abstract}

Objectives: To determine whether cognitive decline in the elderly can be predicted by conducting a psychological assessment.

Study Design: Cohort longitudinal observational study. A community-based comprehensive geriatric assessment (CGA) study for Mild Cognitive Impairment (MCI) was started in March 2015 in Uraus (Hokkaido, Japan). The Tokyo Cognitive Assessment for Mild Cognitive Impairment (ToCAMCI) was administered and cut-off points of $17 / 18$ and 24/25 used to classify 95 elderly citizens in 3 groups. After a 2-year follow-up, the KaplanMeier method was used to test whether the ToCA-MCI score could predict progression to senile dementia.

Results: The frequency of occurrence of dementia was statistically significantly higher in elderly with a ToCA-MCI score of 17 or lower. Such a differentiation was not achieved by the Mini-Mental State Examination (MMSE) recall item.

Conclusions: The short-term memory test of long sentences in ToCA-MCI helped predict progression to dementia. Results prompted the village health nurses to start an education program at the health center, where they provided guidance to increase muscular strength, and nutritional advice.

Highlights: 1. A low ToCA-MCI score can predict progression to senile dementia within 2 years. 2. ToCA-MCI's 25-word story for the delayed recall test helps the prediction. 3. Elderly progression to dementia had a higher pulse wave velocity (PWV). 4. Combined ToCA-MCI and PWV is useful to detect amnestic MCI early in elderly Japanese. 5. ToCA-MCI with PWV cost-effectively predicts dementia progression in poor communities.

Keywords: Dementia, Comprehensive Geriatric Assessment (CGA), Tokyo Cognitive Assessment for Mild Cognitive Impairment (ToCA-MCI), Pulse Wave Velocity (PWV), Education Program

A community-based comprehensive geriatric assessment (CGA) was started in 2000 in a small, poor village, Uraus, in Hokkaido,
Japan, with a population of 937 women and 865 men. Uraus has a full-fledged aged population of $37 \%$. The goal of this investigation 
was to determine how effectively the wellbeing of elderly citizens can be supported with a small investment. After visiting Uraus twice a year for 20 years (from 2000 to 2019) to educate its citizens regarding health promotion, providing every-day life advice on food, exercise and sleep, significant improvement was achieved in terms of blood pressure, diabetes and depressive mood, among others, as reported previously [1-6]. As a result, cost of care for the elderly was lower [7].

The next important challenge was to determine how cognitive decline should be dealt with in the elderly, notably since the cost of specialized equipment, such as brain magnetic resonance imaging, is prohibitive for these villagers. As a solution, based on the Montreal Cognitive Assessment (MoCA), we developed the Tokyo Cognitive Assessment for Mild Cognitive Impairment (ToCA-MCI) [8]. It uses a 25-word story for the delayed recall test in lieu of the 5 words in the MoCA semantic memory test. After the software is installed on a personal computer (PC), citizens reply on the PC to successive questions covering 10 topics. Scoring was automatically calculated for all but 5 subjects, using a cut-off point of $17 / 18$, as previously reported [8].

Since March 2015, we followed-up for 2 years 95 citizens, who were 64 to 95 years of age. The CGA, including ToCA-MCI, was originally applied to 99 elderly, but 4 of them were excluded because 3 already suffered from senile dementia and another had severe hearing impairment. The 95 elderly were classified into 3 groups: Group A consists of 27 citizens (64-88 years, average $75.2 \pm 6.0$ years, 19 females) with a ToCA-MCI score of 25 or higher 5; Group B consists of 38 citizens (68-93 years, average 79.2 \pm 5.5 years, 27 females) with a ToCA-MCI score between 18 and 24; and Group C consists of 30 citizens (65-95 years, average 79.6 \pm 6.2 years, 22 females) with a ToCA-MCI score of 17 or lower. The ToCA-MCI score of the 3 groups averaged $26.4 \pm 1.2,21.5 \pm 2.2$, and $14.2 \pm 3.1$, respectively. The corresponding incidence of senile dementia progression within 2 years was 0,1 , and 7 , respectively.

Results from the Kaplan-Meier analysis are depicted in Figure 1. The frequency of occurrence of dementia was statistically significantly higher in Group C as compared to both Groups B and A. As compared to citizens in Group B, elderly in Group C had a lower score and recalled fewer words of the story regeneration on the short-term memory topic: $1.2 \pm 1.8$ vs. $3.5 \pm 2.3(\mathrm{P}<0.0001)$, and $2.7 \pm 2.3$ vs. $5.8 \pm 3.1$ words $(\mathrm{P}<0.0001)$, respectively. No statistically significant difference was found between them for the Mini-Mental State Examination (MMSE) recall item: $2.10 \pm 0.96$ vs. $2.00 \pm 1.03$ $(\mathrm{P}=0.6841)$, suggesting that it was the short-term memory test of long sentences (25 words) that enabled the prediction of progression to dementia.

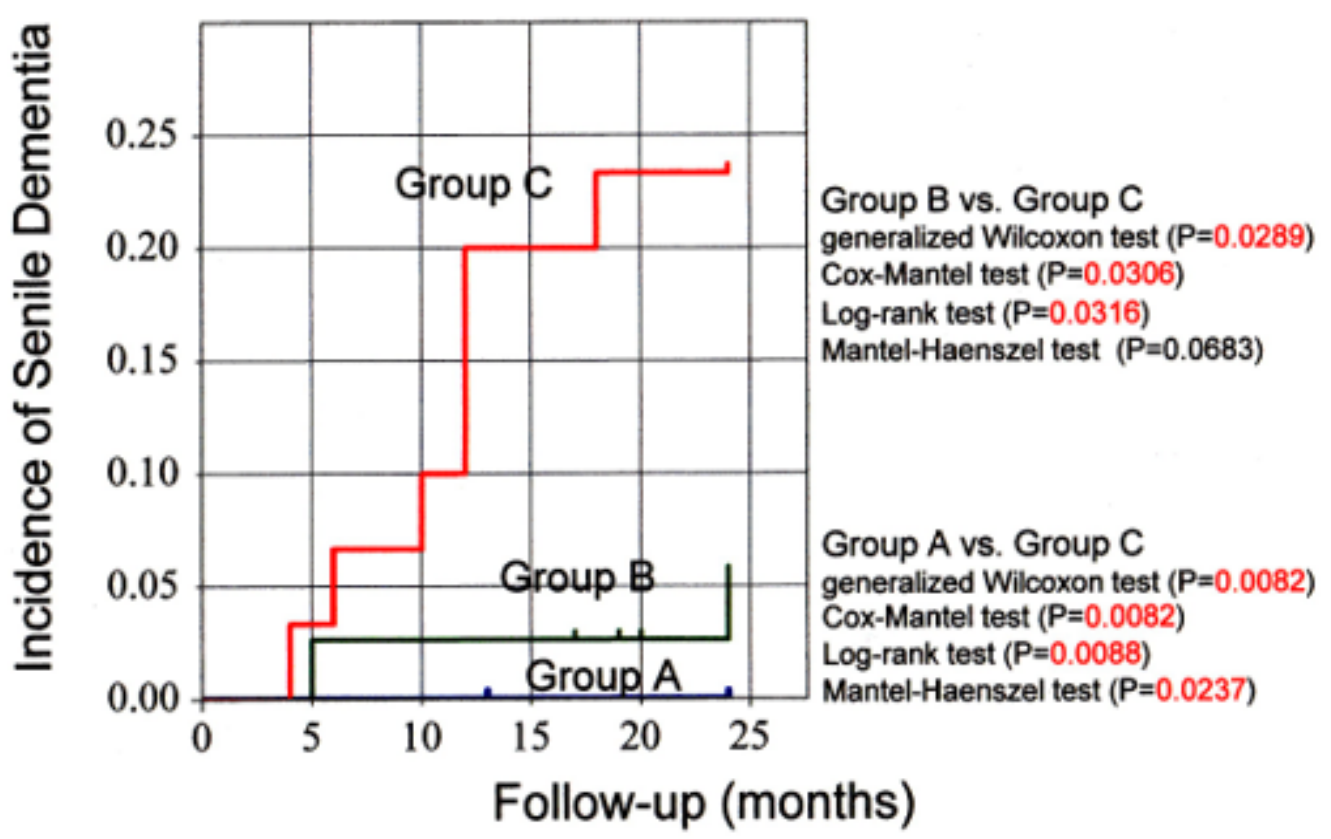

Figure 1: Incidence of senile dementia analyzed by the Kaplan-Meier method.

The occurrence of dementia was statistically significantly higher in Group C (30 citizens; ToCA-MCI score $\leq 17$ ) as compared to Group B (38 citizens; ToCA-MCl score between 18 and 24$)$ by generalized Wilcoxon test $(P=0.0289)$, Cox-Mantel test $(P=0.0306)$ and Log-rank test $(P=0.0316)$, but not by Mantel-Haenszel test $(\mathrm{P}=0.0683)$. It was also statistically significantly higher in Group $\mathrm{C}$ as compared to $\mathrm{Group} \mathrm{A}$ (27 citizens; ToCA-MCI score $\geq 25)$ by generalized Wilcoxon test $(P=0.0082)$, Cox-Mantel test $(P=0.0082)$, Log-rank test $(P=0.0088)$ and Mantel-Haenszel test $(P=0.0237)$. 
In Group C, the 7 citizens who progressed to dementia were older ( $84.7 \pm 6.7$ vs. $78.1 \pm 5.2$ years, $P=0.0465)$, had a quicker pulse (83.4 \pm 4.8 vs. $72.9 \pm 10.0 \mathrm{bpm}, \mathrm{P}=0.0012)$, and a higher pulse wave velocity (PWV) $(2367.6 \pm 224.1$ vs. $1937.1 \pm 383.5 \mathrm{~cm} / \mathrm{s}, \mathrm{P}=0.0021)$ as compared to the other 23 citizens of Group C. As reported previously [9], the combined assessment of ToCA-MCI and PWV is useful for the early detection of amnestic MCI in elderly Japanese. This finding prompted the village's mayor to make available a medical device to measure PWV at the community health center, where citizens can have their PWV measured any time with the help of public health nurses. The 7 citizens who progressed to dementia had a weaker grip strength (15.6 \pm 3.2 vs. $20.9 \pm 6.1 \mathrm{Kg}, \mathrm{P}=0.0075)$ and walked more slowly $(1.123 \pm 0.257$ vs. $1.482 \pm 0.453 \mathrm{~m} / \mathrm{s}, \mathrm{P}=0.0168)$ than the other 23 elderly of group $C$. These results prompted the village health nurses to start an education program at the health center where they provided guidance to increase muscular strength, and nutritional advice. Small trials such as this one may help yield effective outcomes by decreasing morbidity of dementia [10]. Current advances in artificial intelligence technology are rapid, and it may soon be feasible for ToCA-MCI to work fully automatically. It will then be possible to massively administer ToCA-MCI wherever a PC is available and obtain results almost instantly.

\section{Acknowledgements}

The authors thank Ms. Yoshie Saito and the public health nurses from the Health and Welfare Center, Uraus town, Kabato District, Hokkaido, Japan, for cooperation in our study. The authors also acknowledge all elderly participants in the town.

\section{Conflict of Interest}

The authors declare no competing financial and non-financial interests in relation to the work described. There is no sponsor's role.

\section{Author Contributions}

K.O. and G.C. designed the study. K.O. and G.C. wrote the first draft of manuscript and prepared the figure. Y.K., T.K., G.Y. and S.M. analyzed the data and contributed to the writing and editing of the manuscript. All authors read and contributed to the final version of the manuscript.

\section{Ethics}

This study was approved by the Medical Ethics Committee of Tokyo Women's Medical University as Clinical Study \#2912, entitled
"Health assessment of community-dwelling elderly in Japan". Written informed consent was obtained from all participants regarding data analysis and the publication of results thereof.

\section{References}

1. Cornelissen G, Schwartzkopff O, Halberg F, Otsuka K, Watanabe Y, et al. (2001) 7-day ambulatory monitoring for adults with hypertension and diabetes. Am J Kidney Dis 37(4): 878.

2. Otsuka K, Mitsutake G, Yano S (2002) Depression, quality of life, and lifestyle: chronoecological health watch in a community. Biomed Pharmacother 56 (Suppl 2): 231s-242s

3. Cornelissen G, Halberg F, Otsuka K, Singh RB, Chen CH, et al. (2007) Chronobiology predicts actual and proxy outcomes when dipping fails. Hypertension 49 (1): 237-239.

4. Fujisawa M, Ishine M, Okumiya K, Otsuka K, Matsubayashi K, et al. (2007) Trends in diabetes. Lancet 369 (9569): 1257.

5. Kimura Y, Wada T, Ishine M, Ishimoto Y, Kasahara Y, et al. (2009) Food diversity is closely associated with activities of daily living, depression, and quality of life in community-dwelling elderly people. J Am Geriatri Soc 57(5): 922-924.

6. Otsuka K, Cornelissen G, Yamanaka T, Oinuma S, Sasaki J, et al. (2014) Comprehensive geriatric assessment reveals sleep disturbances in community-dwelling elderly adults associated with even slight cognitive decline. J Am Geratric Soc 62(3): 571-573.

7. Matsubayashi K, Wada T, Ishine M, Sakamoto R, Okumiya K, et al. (2010) Community-based geriatric assessment and preventive intervention lowered medical expenses for the elderly. J Am Geriatri Soc 58(4): 791793.

8. Otsuka K, Kubo Y, Yamanaka G, Sasaki J, Kikuchi T, et al. (2017) Tokyo cognitive assessment for mild cognitive impairment, ToCA-MCI. Validity and utility in elderly Japanese. Therapeutic Research 38 (6): 579-621.

9. Otsuka K, Cornelissen G, Yamanaka G, Kubo Y, Kikuchi T, et al. (2019) Combination assessment of ToCA-MCI and Cardio-Ankle Vascular Index (CAVI) is useful for early detection of amnestic MCI in elderly Japanese citizens. In: Orimo $\mathrm{H}$, et al. (Eds.), CAVI and Neuro-Cardiovascular Function ( $\left.1^{\text {st }} e d n\right)$, Vascular Health Promotion Society, Tokyo, Japan, pp. 80-86.

10. Kikuchi T, Okajima K, Cornelissen G, Sasaki J, Oinuma S, et al. (2015) Community-based comprehensive geriatric assessment of shortand long-term predictors of cognitive decline in elderly adults. J Am Geriatrics Soc 63(5): 1031-1033. 